\title{
QuANTITATIVE Subsurface DefEct Detection In Composite Materials USING A NON-CONTACT ULTRASONIC SYSTEM
}

\author{
Donatella Cerniglia \\ Department of Mechanics and Aeronautics, University of Palermo, 90128 Palermo, Italy \\ Boro B. Djordjevic \\ Center for Nondestructive Evaluation, Johns Hopkins University, Baltimore, MD, USA, 21218 \\ Vincenzo Nigrelli \\ DIMSAT, University of Cassino, 03043 Cassino, Italy
}

\begin{abstract}
The results of an experimental study conducted to detect subsurface defects in a thick Gr/PPS composite test sample using a noncontact ultrasonic system are presented. Surface waves are generated by a pulsed laser and detected by an air-coupled capacitance transducer. By controlling the surface wave wavelength through a shadow mask, it is possible to control surface wave penetration depth in the sample. Surface wave peak-to-peak amplitude is related to the near-surface material condition. Results indicate that signal amplitude decreases as the width of the defect increases and an approximately linear relation can be deduced.
\end{abstract}

\section{INTRODUCTION}

Composite structures are being increasingly used in aerospace and other high-technology industries. Defects in such structures can be included during the manufacturing process or can develop during the service life. As a result, reliable nondestructive testing (NDT) techniques are required.

Among the available NDT techniques, contact ultrasonic methods have been largely investigated to assess the integrity of composites both in production and in service [1-2]. The main disadvantages of such methods are the need of a coupling medium and contact requirements.

Non-contact ultrasonic techniques overcome such drawbacks and therefore are receiving growing attention [3-5]. Laser ultrasonics is the most attractive among non-contact NDT techniques. However, laser interferometers as receivers have much lower sensitivity than conventional transducers, especially on poorly reflecting materials as composites. Other alternatives to a completely remote laser-based system are a laser source combined with electromagnetic acoustic transducers (EMATs) or with air-coupled transducers. EMATs, even if technically non-contact, have to be very close to the test object surface and can be used only on electrically conducting surfaces.

A non-contact remote ultrasonic system consisting of a laser source and an air-coupled transducer receiver has been used in [6-8] to detect surface defects in composites using surface waves. These waves have been extensively used to characterize composites [4, 9] and to detect flaws [2]. Surface waves propagate parallel to the solid surface and therefore are extremely suitable to detect nearsurface defects.

In this work the above-mentioned system has been applied pursuing the goal to relate surface wave amplitude to the width of defects. In fact, going beyond the detection of defects, it becomes important to be able to characterize them.

Attenuation of surface waves caused by a defect depends also upon the ratio between the depth of the defect and the wavelength [10], as wavelength is indicative of the penetration depth of surface waves. In fact, their motion is confined, in a quasi-isotropic lay-up, to a layer 


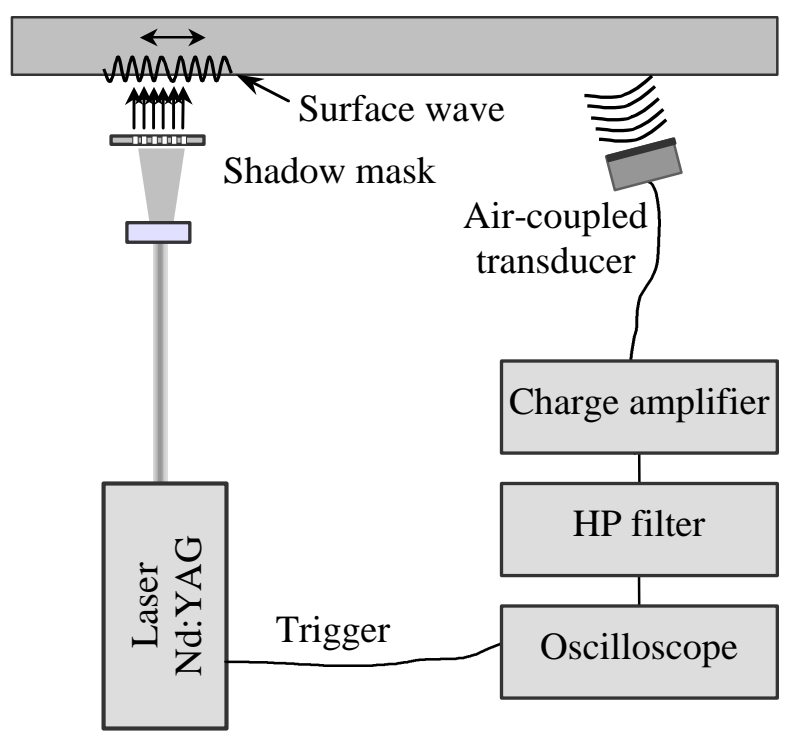

Figure 1 Hybrid ultrasonic test configuration consisting of laser source and air-coupled transducer receiver.

whose thickness is comparable to the wavelength.

The system here used permits control of surface wave wavelength. The generation unit consists of a pulsed laser used in conjunction with a shadow mask. Such mask contains linear slots that - illuminated by the laser beam - project on the sample surface line sources whose spacing represents the surface wave wavelength.

Multi-line sources generation enables also control of surface wave directivity as a line produces highly directional waves [11] and surface waves are generated with the greatest efficiency as total acoustic energy is directed toward the generation of a particular mode of ultrasound.

\section{SETUP AND SAMPLE DESCRIPTION}

The arrangement of the ultrasonic system used in the experiments is illustrated in Fig. 1. The generation unit is a $1064 \mathrm{~nm} \mathrm{Nd:YAG} \mathrm{pulsed}$ laser that produces $10 \mathrm{~ns}$ long pulses at $300 \mathrm{~mJ}$ pulse energy. The shadow mask has six lines and line spacing equal to $2.2 \mathrm{~mm}$. Total energy transmitted across the mask is approximately
$45 \%$ of the incident laser energy. Generation has been realized in thermoelastic regime.

An air-coupled capacitance transducer with an active area $10 \mathrm{~mm}$ in diameter is located in a pitch-catch configuration to detect the surface waves. Orientation angle of the transducer with respect to the sample surface is determined experimentally - for the specific material and the particular direction of propagation - and adjusted at $13.5^{\circ}$, where the surface wave amplitude resulted maximum. Standoff distance along this direction is $5 \mathrm{~mm}$, although bigger air-gap is possible. Distance between the closest line of the mask and the center of the receiver is $103 \mathrm{~mm}$.

The capacitance transducer is connected to a charge amplifier and to a high-pass filter at 0.3 MHz. Output signals are then displayed in an oscilloscope, triggered from the laser. The sample used in the experiments is a consolidated Gr/PPS (LC40-66/AS-4) composite with 560 plies and quasi-isotropic lay-up $\left(0^{\circ},+45^{\circ}, 90^{\circ}\right.$, $45^{\circ}$ ).

Grafoil flaws were intentionally introduced during lay-up at different depths and their position was confirmed by ultrasonic pulse-echo C-scan imaging of the test sample at $2.25 \mathrm{MHz}$ and $5.0 \mathrm{MHz}$.

Figure 2 shows the geometry of the sample and of the defect inspected herein.

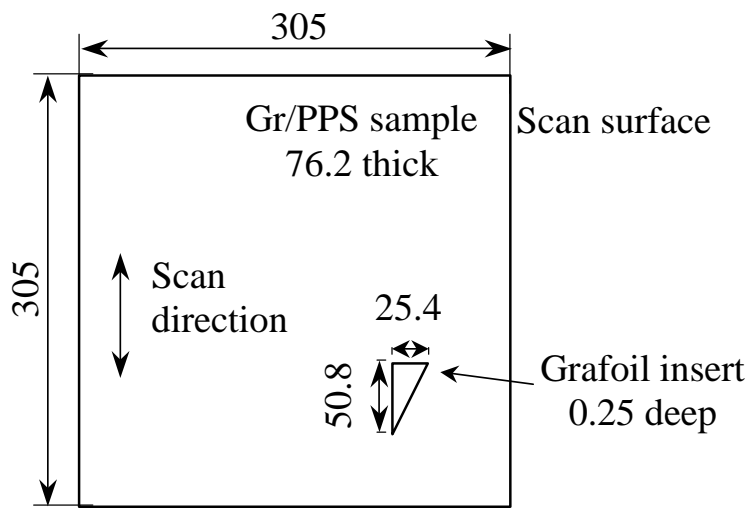

Figure 2 Descriptive geometry of the thick composite sample and of the embedded defect that has been inspected (dimensions in $\mathrm{mm}$ ). 


\section{EXPERIMENTS AND RESULTS}

Tests were performed first in a defect-free area, with the surface wave propagating at $45^{\circ}$ to the fibers direction of the top layer. Figure 3 shows a single-shot waveform acquired in this region. The velocity was measured to be $1.46 \mathrm{~mm} / \mu \mathrm{sec}$ and the frequency $0.65 \mathrm{MHz}$. Surface wave inspection was carried out measuring the amplitude while moving the sample at vertical scan steps of $10 \mathrm{~mm}$.

The scan over the region containing the defect showed that signal amplitude was higher outside the defect whereas dropped to the noise level where defect was wider. Then, as the width of defect decreased the surface wave amplitude increased. Figure 4 shows the signals acquired in the area containing the grafoil insert.

To determine if there is a correlation between width of the defect and surface wave amplitude, the amplitude of the signals acquired is shown in Fig. 5 as a function of the distance on the sample across the defect. In the region with the defect an approximately linear relation between signal amplitude and width of the defect can be deduced.

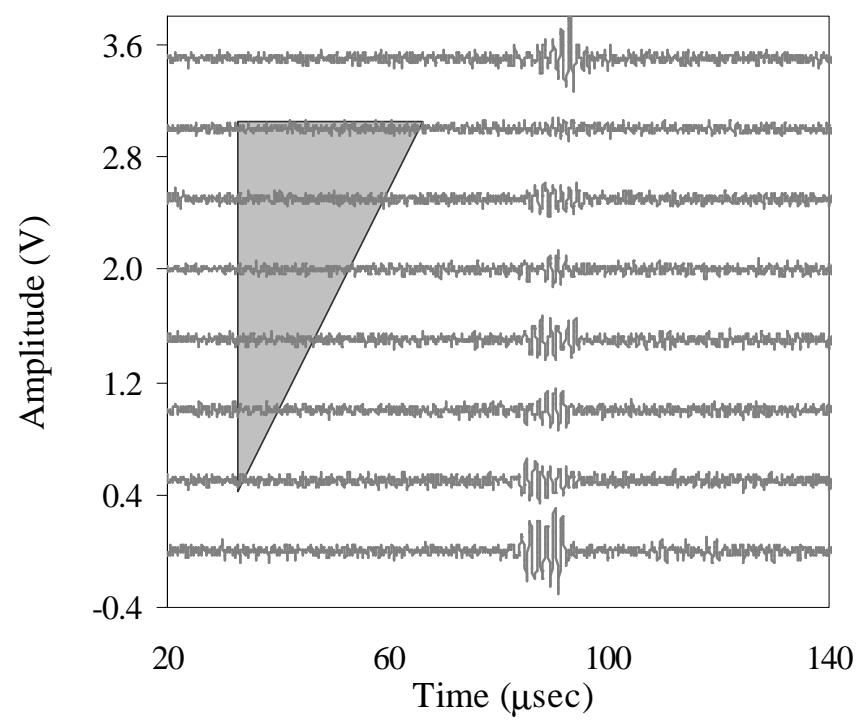

Figure 4 Signals acquired in the area containing the defect. The triangular shape represents the defect.

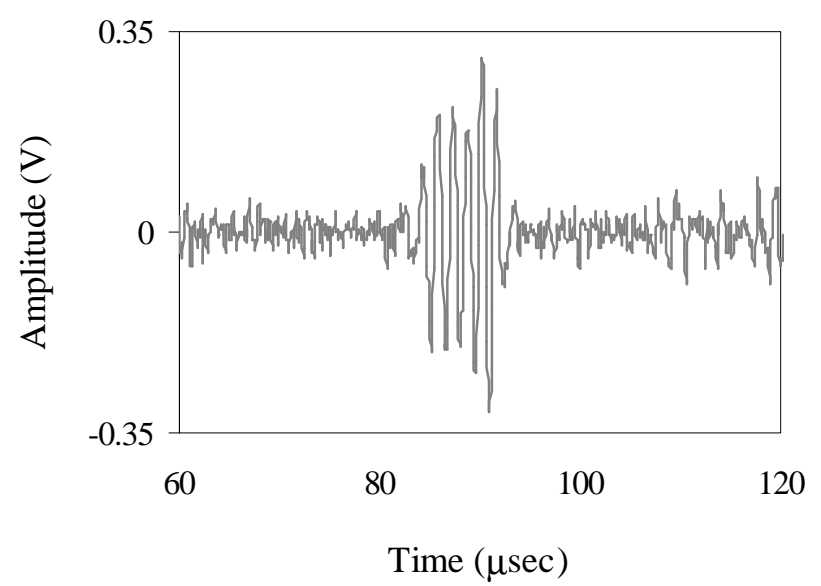

Figure 3 Single-shot surface wave waveform acquired in a defect-free region.

Signals at the two extremities of the defect are acquired with the transducer being not totally over the defect. The active area of the aircoupled transducer, $10 \mathrm{~mm}$ in diameter, affects the results. A smaller aperture is expected to give results more precise, even though with a smaller surface wave amplitude.

Using the above transducer it has been also possible to detect a circular defect and to estimate its size (12 $\mathrm{mm}$ in diameter). A relation between dimensions of the defect and attenuation

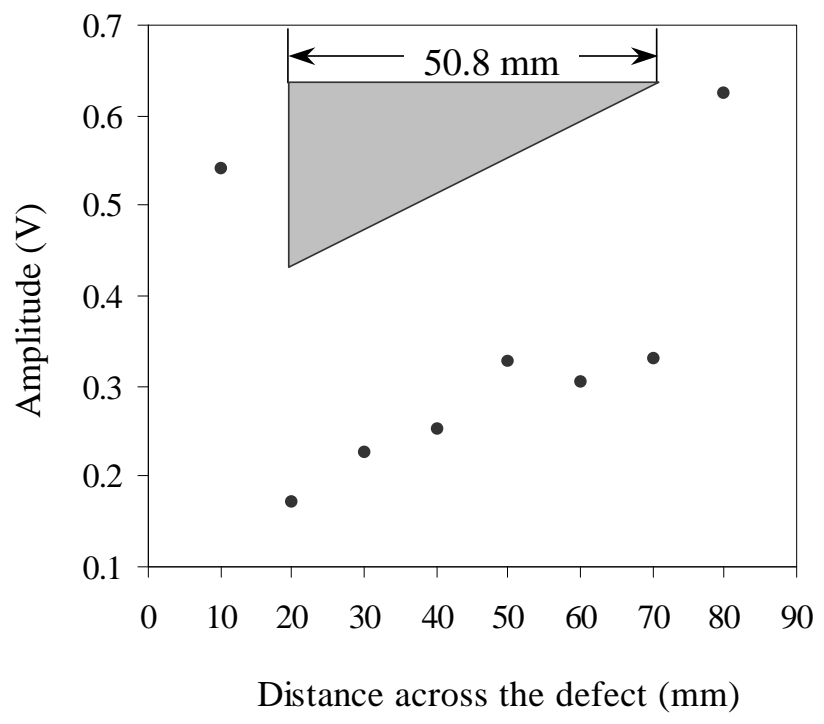

Figure 5 Surface wave peak-to-peak amplitude as a function of the distance on the sample across the defect. 
of the surface wave was not noticeable due both to the size of the transducer and to the shape of the defect.

\section{CONCLUSIONS}

Ultrasonic inspections have been conducted on a thick Gr/PPS composite sample to detect subsurface defects. The surface wave ultrasonic test system consists of a pulsed laser used in conjunction with a shadow mask and of an aircoupled transducer. This system permits control of surface wave wavelength. Surface waves are very suitable for subsurface detection since they penetrate to a depth of about a wavelength in the surface of a quasi-isotropic material. The results show that the system is capable of detecting subsurface defects and indicate a good correlation between surface wave amplitude and width of the defect.

The hybrid ultrasonic system provides easy-tointerpret data and allows inspection of the structure remotely and where access is restricted to one side of the component. Moreover, it does not cause visible damage at the sample surface. As a result, it has potential for extremely rapid evaluation of large areas in a fully automated mode and it could be applied for in-service inspection.

In order to provide significant conclusions additional tests are necessary on composite samples containing different kind of defects.

\section{ACKNOWLEDGEMENTS}

The authors want to thank Uma Bansal for providing help during the experiments.

\section{REFERENCES}

[1] C. Scarponi, and G. Briotti, "Ultrasonic technique for the evaluation of delaminations on CFRP, GFRP, KFRP composite materials", Composites Part B, vol. 31, pp. 237-243, 2000.
[2] S. C. Mantell, and E. J. Linstrom, "Nondestructive evaluation of interlaminar bonding for in situ consolidation of polymer composites", J. Composite Materials, vol. 33, pp. 124-157, 1999.

[3] R. J. Dewhurst, R. He, and Q. Shan, "Defect visualization in carbon fiber composite using laser ultrasound", Mater. Eval., vol. 51, pp. 935-940, 1993.

[4] J. W. Littles, Jr., L. J. Jacobs, and A.-H. Zureick, "Single-sided ultrasonic technique to characterize thick FRP composites", J. Nondestr. Eval., vol. 17, pp. 223-230, 1998.

[5] M. Castaings, P. Cawley, R. Farlow, and G. Hayward, "Single sided inspection of composite materials using air coupled ultrasound", J. Nondestr. Eval., vol. 17, pp. 37-45, 1998.

[6] K. C. Baldwin, T. P. Berndt, and J. Ehrlich, "Narrowband laser generation/air-coupled detection: ultrasonic system for on-line process control of composites", Ultrasonics, vol. 37, pp. 329-334, 1999.

[7] F. Lanza di Scalea, and R. E. Green, "A hybrid non-contact ultrasonic system for sensing bond quality in tow-placed thermoplastic composites", J. Composite Materials, vol. 34, pp. 1860-1880, 2000.

[8] F. Lanza di Scalea, T. P. Berndt, J. B. Spicer, and B. B. Djordjevic, "Remote laser generation of narrow-band surface waves through optical fibers", IEEE Trans. UFFC, vol. 46, pp. 1551-1557, 1999.

[9] J. L. Rose, A. Pilarski, and Y. Huang, "Surface wave utility in composite material characterization", Res. Nondestr. Eval., vol. 1, pp. 247-265, 1990.

[10] C. B. Scruby, and L. E. Drain, Laser Ultrasonics: Techniques and Applications, New York: Adam Hilger, 1990, ch. 6, pp.325-405.

[11] A. M. Aindow, R. J. Dewhurst, and S. B. Palmer, "Laser-generation of directional surface acoustic wave pulses in metals", Optics Comm., vol. 42, pp. 116-120, 1982. 